\title{
Judicious distribution of laser emitters to shape the desired far-field patterns
}

\author{
Constantinos A. Valagiannopoulos and Vassilios Kovanis \\ Department of Physics, School of Science and Technology, Nazarbayev University, 53 Qabanbay Batyr Avenue, \\ Astana KZ-010000, Kazakhstan \\ (Received 4 May 2017; published 6 June 2017)
}

\begin{abstract}
The far-field pattern of a simple one-dimensional laser array of emitters radiating into free space is considered. In the course of investigating the inverse problem for their near fields leading to a target beam form, surprisingly, we found that the result is successful when the matrix of the corresponding linear system is not well scaled. The essence of our numerical observations is captured by an elegant inequality defining the functional range of the optical distance between two neighboring emitters. Our finding can restrict substantially the parametric space of integrated photonic systems and simplify significantly the subsequent optimizations.
\end{abstract}

DOI: 10.1103/PhysRevA.95.063806

\section{INTRODUCTION}

Recently, we have noticed across the photonics literature that there is robust research activity in fabrication, characterization, design, and theoretical developments of large classes of optically interacting oscillators. Examples include (a) arrays of coherently coupled vertical cavity lasers [1], where for the first time the potential of designing the gain-loss profile for non-Hermitian systems was experimentally demonstrated, (b) a network of 37 quantum cascade lasers via antenna mutual coupling [2] which provide a path to increased output power while keeping the intensity of each individual laser low with help from a suitable phase distribution, (c) a network of $64 \times 64$ two-dimensional (2D) large-scale nanophotonic phased arrays employed to produce a great variety of radiation patterns useful in several applications beyond conventional beam focusing and steering [3], (d) fabrication and testing of a large set of silicon optical integrated circuits and implementation of two-dimensional optical beam steering in indium phosphide-based photonic integrated circuits [4], (e) an array of commercially available vertical cavity lasers interacting via diffractive coupling [5], and (f) the manifestation of turbulent chimera states in phased arrays of diode lasers [6]. These photonic circuit implementations are driven by technological applications, such as chip-scale laser radars, short-range optical network communications, next-generation imaging, sensing, and on-demand generation of optically diverse waveforms.

In parallel, we have also recorded substantial theoretical activity in developing an inverse-design paradigm shift in photonic design as it applies to next-generation ultraperforming devices. Based on applied mathematical ideas, the so-called level-set methods for computing moving fronts by Osher [7,8] have been recently translated to photonic devices [9] to the benefit of examining various bounds of solar-cell design and other metamaterial synthetic structures [10]. Prior to the aforementioned recent developments, notable works have also appeared in the framework of topology optimization, including both configuration and materials [11,12]. As far as active structures are concerned, convex optimization has been employed in determining optimal currents that refined the limits for various metrics of radiation performance [13,14]. Similar techniques have been applied to achieve frequencyselective energy transportation with suitable mixtures of active and passive media [15] and for the determination of optimal dielectrics in modeling transformation-optics devices [16]. In particular, a computationally efficient method based on dipole approximation and the reciprocity of space has been introduced by performing an iterative update for both the shape and the texture of the structure under optimization.

In this paper, motivated by such trends in coupled optical oscillators and applied mathematics methods, we inject an inverse-design approach in the construction of the far field of multiple radiating optical apertures. We consider the simple case of a one-dimensional array of equispaced photonic emitters and confine our study to the maximal radiation plane. We do not address how the near fields at the end of the waveguides are produced; instead, we compute the optimal near fields which, via propagation into free space, formulate the desired far-field pattern. Our key mathematical finding is that for a successful pattern reconstitution, the optical distance between two consecutive elements obeys a double inequality. The lower bound expresses the difficulty of reconstructing a rapidly spatially varying pattern with few effective sources. The upper bound is related to the essential spatial coherence between the sources for an efficient beam forming.

This paper is organized as follows. In Sec. II, we present the configuration of an array with several equispaced laser emitters along a line, define the output fields from the waveguides, and state the assumption for the two-dimensional variation. In Sec. III, the far-field pattern is deduced in terms of the local output fields of the lasers by suitably approximating the cylindrical waves emitted from the abruptly terminated waveguides in the far region. In Sec. IV, we find the optimal set of output fields of the lasers to mimic the azimuthal variation of the target pattern, and we present the range that the optical period should belong to in order to have a successful implementation of the proposed method. Finally, in Sec. V, we set as a future target employing the described technique in inverse design of integrated laser systems.

\section{CONFIGURATION AND MOTIVATION}

Typically, a laser comprises a cavity of a finite-length waveguide [17], longitudinally restricted by two mirrors which reflect the light waves and provide feedback to form 


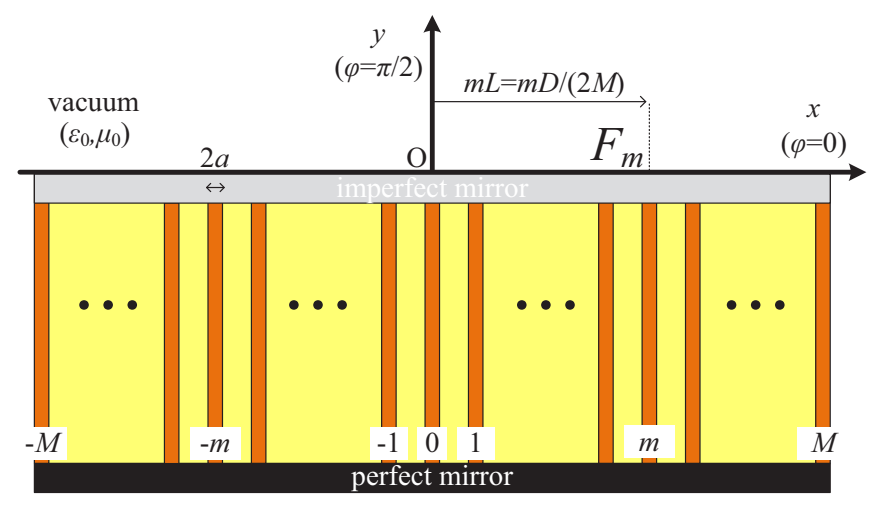

FIG. 1. Schematic of the configuration of the analyzed device. $(2 M+1)$ laser emitters develop independently and locally at their outputs $z$-polarized electric fields $F_{m}$, with $m=-M, \ldots, M$, which propagate into free space. The distance between two consecutive emitters is equal to $L=D /(2 M)$, while the transversal size of each one equals $2 a$. In this way, a far-field pattern $G(\varphi)$ is shaped at $y>0$.

an optical resonator. Into the waveguide, the gain medium gets continuously excited by the circulating electromagnetic field. However, the power in the cavity does not increase unboundedly since one of the two mirrors is not perfect; thus, a certain portion of the signal leaks from the waveguide, which constitutes the output of the device. Very commonly, laser cavities are packed together in order to build coherently or incoherently coupled arrays $[18,19]$ to leverage several beneficial characteristics of the emitting beam (directivity, shaping, power, etc.). Such a laser array is the configuration analyzed in the work at hand.

In particular, we consider the structure schematically depicted in Fig. 1 where $(2 M+1)$ of the aforementioned laser emitters are positioned along the $x$ axis and develop different $z$-polarized electric fields at their outputs, namely, at the position of the imperfect mirror at $y=0$. The complex phasors of the fields are denoted by $F_{m}, m=-M, \ldots, M$, with a suppressed harmonic time $e^{+j \omega t}$. The spacing between two consecutive waveguides is given by $L$, while, with no loss of generality, the transverse size of each of them is taken to be equal to $2 a<L$. The fields $F_{m}$ are considered constant throughout the cross section of each waveguide, which is assumed, again with no loss of generality, to be circular with radius $a$ pegged at $(x, y)=(m L, 0), m=-M, \ldots, M$.

To simplify our analysis, we assume that the phasor of the electric field across the entire zone $\{|x-m L|<a, y=0\}$ equals $F_{m}$; such a reduction renders our problem 2D (field quantities independent of $z$ ). A possible way to achieve this $z$ independence is proposed in Fig. 2: along directions parallel to the $z$ axis, we spatially repeat infinitely many times the same waveguides with identical excitation and therefore identical output $z$-polarized electric fields $F_{m}$. Accordingly, our approach works better for laser arrays located across a strip with length $D=2 M L$ and arbitrary width $W$; in this way, we create an illusion of $z$ independence which gets more successful for larger $W$. We can assume that the distance between two neighboring couples in the $z$ direction is infinitesimal, and thus, a single electric component $\hat{\mathbf{z}} F_{m}$ is developed along the axis $(x, y)=(m L, 0)$. In other words, the outputs of the lasers along

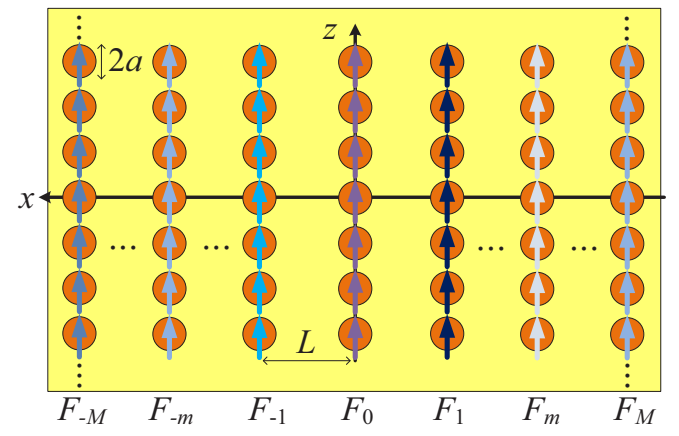

FIG. 2. Our assumption for invariant fields along the $z$ axis can be approximated by considering infinite waveguides terminated at $x=m L$ (and various $z$ ) which produce exactly the same $z$-polarized electric field $F_{m}$ for $m=-M, \ldots, M$. Alternatively, one can consider only $(2 M+1)$ sources with outputs along the line $(x, y)=(0,0)$ and work at the maximal radiation plane $z=0$.

the $x$ axis are uncorrelated to each other, while those along the $y$ direction are the same regardless of the observation point on the imperfect-mirror facet. Equivalently, we can say that we are working with a realistic three-dimensional (3D) configuration of $(2 M+1)$ lasers whose output ports are located along the line $(z, y)=(0,0)$, and we confine our research to the maximal radiation plane $[20] z=0$.

The purpose of this inverse analysis is to determine the local-field sources $F_{m}$ with $m=-M, \ldots, M$, which, by radiation into vacuum, produce an aggregate electric field with a desired azimuthal pattern $\tilde{G}(\varphi)$ in the far region $k_{0} r \rightarrow \infty$. The variables $(r, \varphi)$ are the polar coordinates, which are used interchangeably with $(x, y)$, as indicated in Figs. 1 and 2.

\section{FROM LOCAL FIELDS TO THE FAR-REGION PATTERN}

To quantify the spatial dependence of the electric field $\mathbf{E}(x, y)$ within the vacuum half space $y>0$, we consider the local outputs of the $(2 M+1)$ emitters as displacement current sources or, alternatively, as radiating apertures. Furthermore, we can ignore the structure of the cavity lasers' array at $y<0$ and replace them with free space; their job was just to provide us with the local fields $F_{m}$, which have already been taken into account. In this sense, we can work as if $(2 M+1)$ sources radiate simultaneously into free space (for both $y>0$ and $y<0$ ). We expect that the actual field for $y>0$ will be well approximated by the aggregate field of the aforementioned sources, especially in the far region.

It is straightforward [21,22] to show that the $z$ electric component developed by the $m$ th source alone in free space is given by

$$
E_{m}(x, y)=\frac{F_{m}}{H_{0}^{(2)}\left(k_{0} a\right)} H_{0}^{(2)}\left(k_{0} \sqrt{(x-m L)^{2}+y^{2}}\right),
$$

where $k_{0}$ is the wave number of free space and $H_{0}^{(2)}$ is the Hankel function of the zeroth order and second type (the solution to the scalar wave equation in cylindrical or polar coordinates [21,23]). If one uses the polar coordinate system $(r, \varphi)$, one finds that the total $z$-polarized field 
$E=\sum_{m=-M}^{M} E_{m}$ of all the considered sources in free space takes the form

$$
E(r, \varphi)=\frac{1}{H_{0}^{(2)}\left(k_{0} a\right)} \sum_{m=-M}^{M} F_{m} H_{0}^{(2)}\left(k_{0} r A_{m}(r)\right),
$$

where $A_{m}(r)=\sqrt{1-2 \frac{m L}{r} \cos \varphi+\left(\frac{m L}{r}\right)^{2}}$. In the far region $\left(k_{0} r \rightarrow \infty\right)$, we can find an approximate equivalent if we utilize the asymptotic expansion of the Hankel function for large arguments [24]:

$$
H_{0}^{(2)}\left(k_{0} R\right) \sim \sqrt{\frac{2 j}{\pi k_{0} R}} \exp \left(-j k_{0} R\right), \quad k_{0} R \rightarrow \infty .
$$

In other words, the Hankel function far from the source behaves as a cylindrical wave with the amplitude vanishing with the square root of the optical distance $k_{0} R$. If one additionally makes the approximation $A_{m}(r) \cong 1-\frac{m L}{r} \cos \varphi$ for the phase in the far region $r \gg M L$, the electric far field produced by the array of lasers at $y>0$ is written as

$$
\begin{aligned}
E(r, \varphi) & \cong \frac{e^{-j k_{0} r}}{H_{0}^{(2)}\left(k_{0} a\right)} \sqrt{\frac{2 j}{\pi k_{0} r}} \sum_{m=-M}^{M} F_{m} e^{j k_{0} L m \cos \varphi} \\
& \equiv \sqrt{\frac{2 j}{\pi k_{0} r}} e^{-j k_{0} r} G(\varphi) .
\end{aligned}
$$

For the amplitude, we perform the less sharp [21] approximation: $A_{m}(r) \cong 1$ when $k_{0} r \rightarrow \infty$. Also the cylindrical (dimensionless) propagation factor $\sqrt{\frac{2 j}{\pi k_{0} r}} e^{-j k_{0} r}$, which is common for the far field of every finite-size source [25], is dropped for brevity.

Therefore, we have an explicit expression for the azimuthal profile of the far-field $G(\varphi)=\sum_{m=-M}^{M} \frac{F_{m}}{H_{0}^{(2)}\left(k_{0} a\right)} e^{j k_{0} L m \cos \varphi}$ (measured in volts/meter), produced by $(2 M+1)$ infinite series of coupled cavity lasers with local electric fields $F_{m}$ (measured in volts/meter) for $m=-M, \ldots, M$.

The aforementioned analysis would also be valid for the 3D problem of a single set of $(2 M+1)$ laser emitters located along the $x$ axis provided that we care only about the field distribution on the maximal radiation plane $(z=0)$. Each of the $(2 M+1)$ waveguides is characterized by the $z$-polarized electric field $F_{m}$, which is supposed to be homogeneous in the volume of a small sphere of radius $a$ centralized at $(x, y)=(0, m L)$ and constitutes the end of the corresponding ( $m$ th) waveguide $(m=-M, \ldots, M)$. For observation points positioned on the $x y$ plane, the electric field developed due to the $m$ th laser has a sole $z$ component with the complex phasor $E_{m}(r, \varphi)=$ $F_{m} \frac{k_{0} a}{e^{-j k_{0} a}} \frac{e^{-j k_{0} r A_{m}(r)}}{k_{0} r A_{m}(r)}$. Note that, since we are referring to the slice $z=0$ of our $3 \mathrm{D}$ space, the distance of an observation point from the $z$ axis (cylindrical radial coordinate $r$ ) is the same as its distance from the origin (spherical radial coordinate).

In this way, one can obtain an expression for the aggregate far field that is almost the same as $(4): E(r, \varphi) \cong$ $\frac{k_{0} a}{e^{-j k_{0} a}} \frac{e^{-j k_{0} r}}{k_{0} r} \sum_{m=-M}^{M} F_{m} e^{j k_{0} L m \cos \varphi}$. In fact, we realize that the only difference from (4) is the $r$ dependence, describing the field of a spherical source, which expresses the inevitable attenuation because of the distribution of power around larger and larger semicircles of radius $r$. However, this common factor $\frac{e^{-j k_{0} r}}{k_{0} r}$ does not play a part in our inverse problem; we count only the $\varphi$-dependent pattern $G(\varphi)=\frac{k_{0} a}{e^{-j k_{0} a}} \sum_{m=-M}^{M} F_{m} e^{j k_{0} L m \cos \varphi}$. Therefore, the approach of making the variational sum $G(\varphi)$ as similar as possible to the target pattern $\tilde{G}(\varphi)$, which is the objective of Sec. IV, remains unaltered whether we consider the $2 \mathrm{D}$ problem or the special case of the 3D one.

\section{FROM THE DESIRED FAR-REGION PATTERN TO OPTIMAL LOCAL FIELDS}

Ideally, we should aim at achieving the exact equality between the obtained field pattern $G(\varphi)$ created by the lasers and the target (desired) pattern $\tilde{G}(\varphi)$ for all angles $0<\varphi<$ $180^{\circ}$ of the upper half space $y>0$. However, this is not always possible, and in order to develop a general technique which can be applied to any variation of $\tilde{G}(\varphi)$, we should search for the optimal set of output fields $\left\{F_{m}, m=-M, \ldots, M\right\}$ best mimicking the desired pattern.

We notice that $G(\varphi)$ is expressed as a finite sum of the basis functions set: $\left\{p_{m}(\varphi)=e^{j k_{0} L m \cos \varphi}, m=-M, \ldots, M\right\}$. These functions do not constitute a complete set due to the finite size $(2 M+1)$ of their population. Furthermore, the variations of $p_{m}(\varphi)$ are dependent on the parameter $k_{0} L$, which expresses how sparse our emitter lattice is. Choosing a small $k_{0} L$ for fixed $M$ will make us lose the ability to imitate rapid $\varphi$ variations; on the other hand, choosing a large $k_{0} L$ will decrease our "resolution" of mimicking patterns since only specific waveforms, very different from each other, would be possible to produce. Therefore, a careful study of the degrees of azimuthal variational freedom and numerical robustness of our method with respect to $k_{0} L$ is required.

As far as the orthogonality of the basis-function set $\left\{p_{m}(\varphi), m=-M, \cdots, M\right\}$ is concerned, it does not exist either (the same as the completeness property). In particular, the internal product of two members of the set over the azimuthal range $0<\varphi<180^{\circ}$ is nonzero; however, it can be analytically [24] evaluated:

$$
\begin{aligned}
\frac{1}{\pi} \int_{0}^{\pi} p_{m}(\varphi) p_{n}^{*}(\varphi) d \varphi & =\frac{1}{\pi} \int_{0}^{\pi} \exp \left[j k_{0} L(m-n) \cos \varphi\right] d \varphi \\
& =J_{0}\left(k_{0} L(m-n)\right) \equiv S_{n m}
\end{aligned}
$$

where $m, n=-M, \ldots, M$ and $J_{0}$ is the zeroth-order Bessel function. If we try to project the ideal equality $G(\varphi)=\tilde{G}(\varphi)$ on the conjugate (*) set of basis functions, namely, adopt the Galerkin [26] approach (where the testing functions are the complex conjugate of the basis functions), we obtain the $(2 M+1) \times(2 M+1)$ linear system $\mathbf{S} \cdot \mathbf{f}=\mathbf{v}$ with unknown optimal vector of fields $\mathbf{f}=\left[F_{-M} \cdots F_{M}\right]^{T}$. The matrix of the system $\mathbf{S}=\left[S_{n m}\right]$ contains solely values of function $J_{0}$ as indicated by (5), which appears in a number of intriguing physical situations [27,28]. Note that $\mathbf{S}$ is real and symmetric and its diagonal elements are all equal to $1: J_{0}(0)=1$. When it comes to the constant vector $\mathbf{v}=\left[V_{-M} \cdots V_{M}\right]^{T}$, it is defined by the following general term:

$$
V_{n}=\frac{H_{0}^{(2)}\left(k_{0} a\right)}{\pi} \int_{0}^{\pi} \tilde{G}(\varphi) \exp \left(-j k_{0} L n \cos \varphi\right) d \varphi,
$$




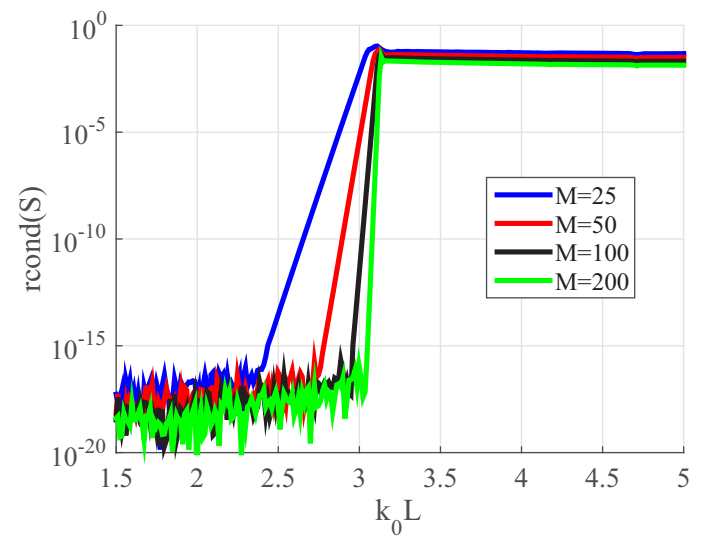

(a)

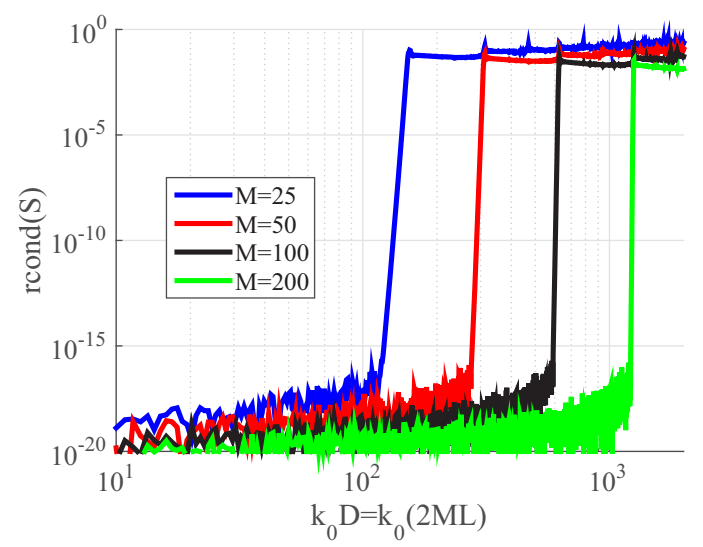

(b)

FIG. 3. The reciprocal condition number of our linear system matrix $\mathbf{S}$ as function of (a) the optical period of our laser lattice $k_{0} L$ and (b) the optical size of our entire cluster of lasers $k_{0} D=k_{0}(2 M L)$ for several numbers of lasers $M$.

which contains the components of the ideal pattern $\tilde{G}(\varphi)$ in the basis of the testing-function set: $\left\{p_{n}^{*}(\varphi)=e^{-j k_{0} L n \cos \varphi}, n=\right.$ $-M, \ldots, M\}$.

Since the numerical behavior of the matrix $\mathbf{S}$ determines the accuracy and robustness of the linear system with respect to $\mathbf{f}$, let us examine the reciprocal condition number rcond of S. Such a number [29] returns an estimate for the reciprocal condition of $\mathbf{S}$ in the 1-norm [S is well conditioned for $\operatorname{rcond}(\mathbf{S}) \rightarrow 1^{-}$and $\mathbf{S}$ is badly scaled for $\operatorname{rcond}(\mathbf{S}) \rightarrow 0^{+}$]. In Fig. 3(a), we show $0<\operatorname{rcond}(\mathbf{S})<1$ as a function of the optical period of the laser lattice $k_{0} L$ for various populations $M$ of lasers. The curves corresponding to $M=25$ and $M=50$ are shown in blue (dark gray) and red (medium gray), respectively, while the data for $M=100$ and $M=200$ are depicted in black and green (light gray), respectively. One clearly notices that our numerical inversion is more accurate for larger $k_{0} L$. This conclusion may be anticipated since the variation of $J_{0}\left(k_{0} R\right)$ resembles a cosine with decaying amplitude [such as $1 / \sqrt{\left|k_{0} R\right|}$ [24] from (3)]; therefore, for a high $k_{0} L$ all the off-diagonal elements of the matrix $\mathbf{S}$ would be small compared to its unitary diagonal ones, and $\mathbf{S}$ would behave robustly as a close-to-diagonal matrix. It should be stressed that the number of lasers $M$ does not play a crucial role in the numerical behavior of $\mathbf{S}$; in all cases, regardless of $M$, the matrix $\mathbf{S}$ acquires a good condition approximately for $k_{0} L>3$. By increasing the number of lasers, one just makes the overall device longer. However, in Fig. 3(b), where $\operatorname{rcond}(\mathbf{S})$ is represented as a function of the total size of our laser cluster $k_{0} D=k_{0}(2 M L)$, we can apparently observe that sparser structures (smaller $M / k_{0} D$ ) exhibit better numerical behavior.

But would just utilizing a long array with large optical distances $k_{0} L$ between two neighboring lasers be the correct choice? Certainly, based on Fig. 3, this array will give a very low error for the linear system $\|\mathbf{S} \cdot \mathbf{f}-\mathbf{v}\|$, that is, will return fields $\mathbf{f}$ satisfying exactly the projection equations. But what is happening with the actual error $\int_{0}^{\pi}|\tilde{G}(\varphi)-G(\varphi)| d \varphi$ between the target pattern $\tilde{G}(\varphi)$ and the produced far-field $G(\varphi)$, which is the crucial quantity determining the degree of success of our approach? As indicated above, a large $k_{0} L$ will substantially reduce the ability of the variational finite sum $G(\varphi)$ to mimic the desired response $\tilde{G}(\varphi)$. For this reason, we should balance the demand for high $k_{0} L$, ensuring stable numerical behavior (small linear system error), and the requirement of low $k_{0} L$ (accompanied by suitably large $M$ ), leading to a significant capacity for $\varphi$-variation adjustment (small deviation between the actual $G$ and ideal target $\tilde{G}$ pattern).

To test the efficiency of our process, let us consider the following family of desired patterns $\tilde{G}(\varphi)$ :

$$
\tilde{G}(\varphi)=\exp (-\beta \varphi)[1+A \cos (\alpha \varphi)]
$$

where the magnitude $A$ expresses the difference of $\tilde{G}(\varphi)$ from an omnidirectional pattern, the number $\alpha$ determines the rapidness of oscillations with respect to $\varphi$, and the quantity $\beta$ specifies the envelope trend. It is a demanding and "unnatural" formula which contains several types of azimuthal variations; if our method performs well with such a not easily satisfied target, it will perform well with more natural beam shapes.

In Fig. 4, we represent with the solid red (light gray) line an ideal target $\tilde{G}(\varphi)$ from (7) with $A=0.7, \alpha=13.5, \beta=0.2$ for $M=50$, and we apply the technique described above to obtain actual patterns $G(\varphi)$ for various optical distances between the emitters $k_{0} L$. The real part of $G(\varphi)$ is shown as thick curves made of consecutive circles, while the imaginary part usually has a small magnitude and is sketched with black curves close to the horizontal $\varphi$ axis. In Fig. 4(a), where a very tiny $k_{0} L$ is selected, our method totally fails: the real parts differ substantially. Such a poor result is not due to the fact that the matrix $\mathbf{S}$ of the system is badly conditioned but mainly because the describing set of functions $\left\{p_{m}(\varphi)=\right.$ $\left.e^{j k_{0} L m \cos \varphi}, m=-M, \ldots, M\right\}$ does not have components with $\varphi$ variation rapid enough to mimic the waveform $\tilde{G}(\varphi)$. Indeed, the maximally varying (with respect to $\varphi$ ) basis function from the aforementioned set is the one corresponding to $m=$ $M: p_{M}(\varphi)=e^{j k_{0} L M \cos \varphi}=e^{j k_{0} D \cos \varphi / 2}$. The most rapid azimuthal variation of this function happens around $\varphi=0^{\circ}$ and $\varphi=180^{\circ}$ since there the argument of the harmonic exponential gets maximized. In the vicinity of such angles, we obtain $|\cos \varphi| \cong 1$. Therefore, the degree $u$ of the largest significant harmonic $e^{+j u \varphi}$ contained in $p_{M}(\varphi)$ (and, accordingly, in the entire set of basis functions) is approximately 


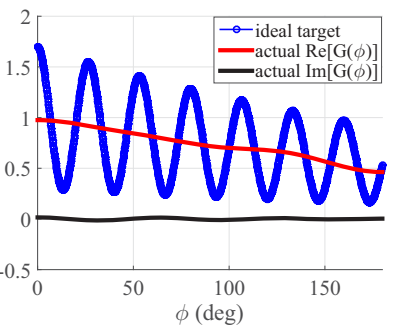

(a)

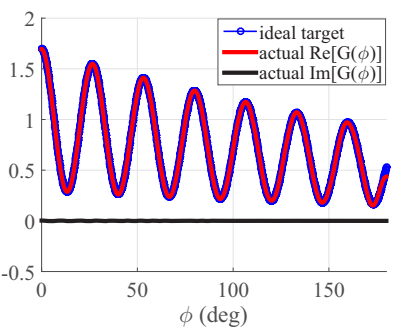

(c)

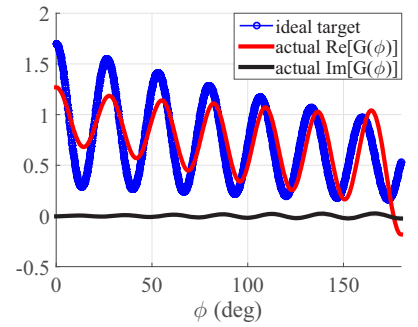

(b)

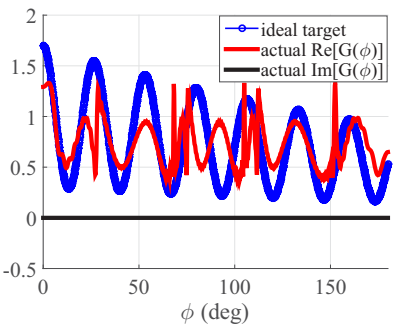

(d)
FIG. 4. The ideal target $\tilde{G}(\varphi)$ and the optimal actual pattern $G(\varphi)$ (both real and imaginary parts) as functions of azimuthal angle $\varphi$ for (a) $k_{0} L=0.005$, (b) $k_{0} L=0.1$, (c) $k_{0} L=1$, and (d) $k_{0} L=10$. Plot parameters: $A=0.7, \alpha=13.5, \beta=0.2$, referring to (7), and $M=50$.

$u=\left\lfloor k_{0} D / 2\right\rfloor=M\left\lfloor k_{0} L\right\rfloor$. In other words, if we have a desired target with rapid azimuthal variation dictated by a maximum significant harmonic $u_{\max }>u=M\left\lfloor k_{0} L\right\rfloor$, then it is not possible for the set of functions $\left\{p_{m}(\varphi)=e^{j k_{0} L m \cos \varphi}, m=\right.$ $-M, \ldots, M\}$ to capture its waveform. This is the case in Fig. 4(a).

If we select a higher $k_{0} L$ as in Fig. 4(b), again, the obtained pattern $G$ is substantially different from the desired $\tilde{G}$, and also a significant imaginary part for $G(\varphi)$ is obtained (instead of zero). Once more, the difference is not attributed to the numerical inversion as an outcome of an unstable matrix $\mathbf{S}$ since the error $\|\mathbf{S} \cdot \mathbf{f}-\mathbf{v}\|$ is negligible; the problem is again that $k_{0} L<u_{\max } / M$, as in Fig. 4(a). Therefore, we realize that the small $\operatorname{rcond}(\mathbf{S})$ indicated in Fig. 3 is not an issue; unless we are talking about an extremely badly conditioned matrix, it can be numerically inverted with negligible numerical error in a MATLAB environment. Despite the fact that a warning message appears at the MATLAB command line [automatically activated when $\operatorname{rcond}(\mathbf{S})$ falls below a specific threshold], the computational platform has sophisticated toolboxes with adaptive algorithms and transformations which can handle well the cases of nonrobust systems $\mathbf{S} \cdot \mathbf{f}=\mathbf{v}$. The symmetric nature of our matrix $\mathbf{S}$ definitely contributes to this direction [30-32].

In contrast to Figs. 4(a) and 4(b), in Fig. 4(c), the described method works perfectly, and the variation of $\tilde{G}(\varphi)$ is almost flawlessly captured with negligible error (in both the real and imaginary parts). However, as is obvious from Fig. 3(a), the matrix of the system $\mathbf{S}$ continues to be close to singular, which is another indication that it does not damage the efficiency of the proposed technique. Of course, such a badly scaled matrix will have an impact on the magnitude of the unknown vector $\mathbf{f}$, namely, the field amplitudes $\left\{F_{m}, m=-M, \ldots, M\right\}$; nevertheless, we do not care much about it as we seek fields and waveforms normalized by their maximal value (relative results).

In Fig. 4(d), we increase further the optical period of the array of our lasers, and we surprisingly realize that our method fails. The reason this time is the low-resolution potential of the describing set of functions originating from the fact that its members are very different from each other, which means that they can mimic successfully only a few isolated patterns (not a continuous area in pattern space). Empirically, we notice that such a harmful effect happens when the matrix $\mathbf{S}$ is well scaled! In other words, a linear system very numerically robust [with large $\operatorname{rcond}(\mathbf{S})$ ] is accompanied by a totally inefficient set of basis functions [a substantial difference between the actual pattern $G(\varphi)$ and the target pattern $\tilde{G}(\varphi)$ ]. To avoid such a failure, we should select $k_{0} L$ less than 3 , as indicated by Fig. 3(a).

If one combines the two aforementioned restrictions for $k_{0} L$, one can obtain the following rule of thumb:

$$
\frac{u_{\max }}{M} \lesssim k_{0} L \lesssim 3
$$

for a fixed number of $(2 M+1)$ available lasers and a desired pattern $\tilde{G}(\varphi)$ with maximal significant azimuthal component $e^{ \pm j u_{\max } \varphi}$. If $u_{\max } / M>3 \Rightarrow M<3 u_{\max }$, then we do not have enough lasers to mimic such a rapidly oscillating far field. Inequality (8) is the key mathematical conclusion of this work.

The elegant, although empirical and approximate, double inequality (8) can be additionally verified if we pose a more realistic target pattern describing tilted directive beams. In particular, let us consider

$$
\tilde{G}(\varphi)=\exp \left[-\gamma(\varphi-\vartheta)^{2}\right]
$$

where $\gamma>0$ determines how directive the main lobe of the radiation pattern would be around angle $\varphi=\vartheta$. As in the target pattern of (7), $2 \pi$ periodicity is not required since the regarded area $\left(0<\varphi<180^{\circ}\right)$ is not a complete circle. We can directly express (the even expansion of) $\tilde{G}$ as a Fourier series; namely, $\tilde{G}(\varphi)=\sum_{u=0}^{+\infty} C_{u} \cos (u \varphi)$. In order to estimate the maximal significant azimuthal order $u_{\max }$, we represent [Fig. 5(a)] the magnitudes of the Fourier coefficients $\left|C_{u}\right|$ as a function of $u$ for various parameters $\gamma$ of (9), while we keep constant the maximal radiation at $\vartheta=90^{\circ}$. It is clear that $C_{u}=0$ for odd $u$, and the solid curves are only guides to the eye. Given the fact that a substantial $\gamma$ corresponds to narrow beams, it is natural that more Fourier coefficients are required in order to approximate the waveforms at a satisfying degree; therefore, $u_{\max }$ increases with $\gamma$. In Figs 5(a)-5(d), we try to mimic the far-field response for each of the aforementioned three cases with a specific set of lasers $(M=80)$ and fixed placement $\left(k_{0} L=0.1\right)$. The color scheme is the same as in Fig. 4. It is obvious that the optimal output fields calculated by the proposed method make an excellent reproduction of $\tilde{G}(\varphi)$ for $\gamma=10$ [Fig. 5(b)]. However, a small error between $\tilde{G}(\varphi)$ and $G(\varphi)$ is recorded for the more directive beam of $\gamma=40$ [Fig. 5(c)], whose azimuthal variation is more abrupt. Finally, our approach does not perform well for the steepest beam case [Fig. 5(d)], where spurious sidelobes appear and the main maximum is substantially weakened. We can draw the conclusion that since $u_{\max }$ is the only quantity that changes in Figs. 5(b)-5(d), we have $u_{\max }<M k_{0} L$ in the first two cases 


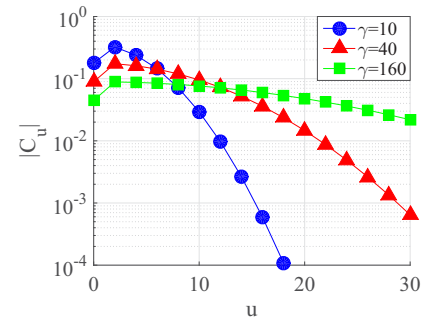

(a)

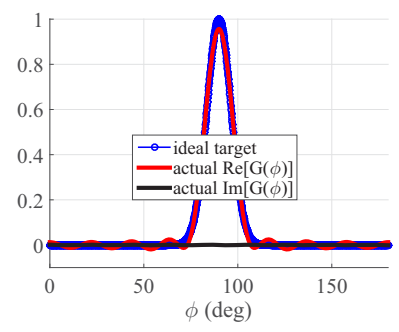

(c)

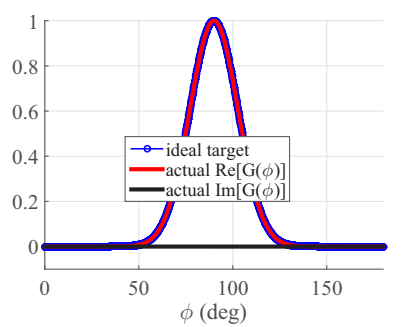

(b)

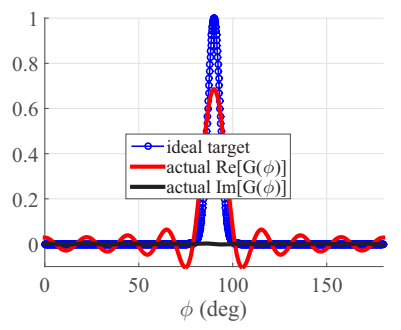

(d)

FIG. 5. (a) The magnitude of the Fourier coefficients $\left|C_{u}\right|$ as a function of the azimuthal order $u$ for various target patterns, referring to (9). Solid curves are only guides to the eye, and odd-ordered coefficients are identically zero. The ideal target $\tilde{G}(\varphi)$ and the optimal actual pattern $G(\varphi)$ (both real and imaginary parts) are shown as functions of azimuthal angle $\varphi$ for (b) $\gamma=10$, (c) $\gamma=40$, and (d) $\gamma=160$. Plot parameters: $\vartheta=90^{\circ}$, referring to (9), $k_{0} L=0.1$, and $M=80$.

[ $\gamma=10,40$ in Figs 5(a) and 5(c)] and $u_{\max }>M k_{0} L$ in the last one $[\gamma=160$ in Fig. 5(d)]. It is important to stress that these conditions are approximate to the same degree as the maximum significant azimuthal order $u_{\max }$ and both inequalities of the rule of thumb (8) are also roughly evaluated. That is why we expressed the double inequality (8) in terms of $\lesssim$ symbols.

In Fig. 6, we show how the emitter array used in Fig. 5 performs when the target pattern of (9) is quite directive $(\gamma=40)$ and tilted with various tilt angles $\vartheta$. The same color scheme as in Fig. 4 applies. The only difference between the systems producing the responses in Fig. 6 is the fields $F_{m}, m=-M, \ldots, M$, which are obtained by inverting the aforementioned linear system $\mathbf{S} \cdot \mathbf{f}=\mathbf{v}$ with different constant vectors $\mathbf{v}$ computed from (6). It is remarkable that the performance of our method deteriorates when the target beam becomes less parallel to the interface $y=0$ (smaller angle $\vartheta$ ) despite the fact that $u_{\max }$ is similar in all four considered scenarios. To put it another way, the approximate inequality (8) defining the range of lattice period $k_{0} L$ for successful implementation of the described technique obviously does not take into account all the parameters of the considered problem. A tilted target radiation pattern $\tilde{G}(\varphi)$ contains an additional inherent difficulty since it forces stronger output fields from the emitters close to the one end of the array. Such an imbalance in the distribution of the optimal fields $F_{m}$ with $m=-M, \ldots, M$ along the lasers at $y=0$ leads to a less accurate copy of the target pattern; naturally, the highest performance is recorded for $\vartheta=90^{\circ}$ as in Fig. 5(c). Note that the depicted results are not the best we can achieve with the proposed method; in both Figs. 5 and 6, we keep the optical distance between two consecutive lasers fixed (and quite small): $k_{0} L=0.1$.

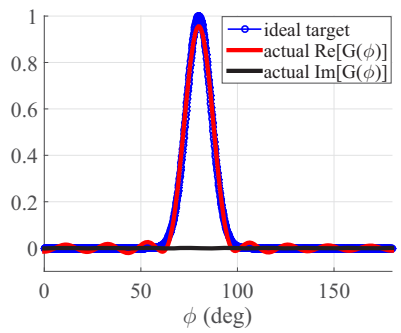

(a)

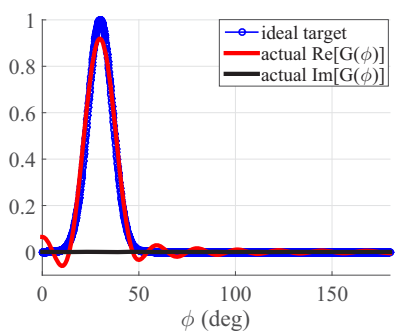

(c)

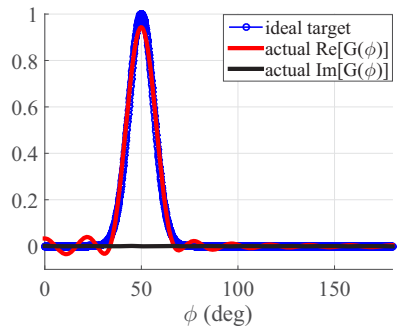

(b)

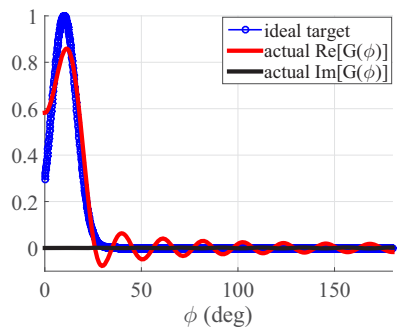

(d)
FIG. 6. The ideal target $\tilde{G}(\varphi)$ and the optimal actual pattern $G(\varphi)$ (both real and imaginary parts) as functions of azimuthal angle $\varphi$ for (a) $\vartheta=80^{\circ}$, (b) $\vartheta=50^{\circ}$, (c) $\vartheta=30^{\circ}$, and (d) $\vartheta=10^{\circ}$. Each optimal pattern corresponds to a different set of output fields dictated by the target pattern. Plot parameters: $\gamma=40$, referring to (9), $k_{0} L=0.1$, and $M=80$.

We did not calibrate this parameter in order to obtain perfect results as in Fig. 4(c) since our intention was to demonstrate the validity of (8) and express clearly the limitations of the presented concept when more and more challenging targets are posed.

\section{CONCLUSIONS AND FUTURE WORK}

In this work, we presented an inverse method of picking (by solving a linear system) the optimal local fields at the outputs of a periodic laser array in order to make a desired far-field pattern. We concluded that the quality of the reconstruction of a given beam is directly related to the distance between two emitters. For this reason, we obtained an elegant inequality that suggests a safe range for the crucial parameter that we consider. It is remarkable that for the successful implementation of the proposed technique, it is not necessary to have a wellconditioned matrix; the numerical inversion is carried out without stability issues due to certain advantageous features of the matrix (it is real, symmetric, and defined solely through a zeroth-order Bessel function).

In our analysis, we have assumed that creating a specific set of local fields at the ends of the laser waveguides is always feasible. However, such a result is related to the characteristics of the waveguides (length, cavity coupling), the employed materials (gain, nonlinearities), and the current driving (injection, sources) [33]. A significant step forward would be to formulate the inverse problem not with respect to the outputs of the emitters (which is done in this work) but with respect to the geometrical, material, and power features of the laser arrays which produce a far-field target response. 
Another very interesting continuation of the present work would be to consider the problem of inversely developing a far-field pattern dependent not only on the azimuthal angle but also on the zenith angle of spherical coordinates. This would require a two-dimensional array of emitters, and the electric-field pattern would possess two components normal to the radial direction.

[1] A. Gao, S. T. M. Fryslie, B. J. Thompson, P. S. Carney, and K. D. Choquette, Parity-time symmetry in coherently coupled vertical cavity laser arrays, Optica 4, 323 (2017).

[2] T. Y. Kao, J. L. Reno, and Q. Hu, Phase-locked laser arrays through global antenna mutual coupling, Nat. Photonics 10, 541 (2016).

[3] J. Sun, E. Timurdogan, A. Yaacobi, E. S. Hosseini, and M. R. Watts, Large-scale nanophotonic phased array, Nature (London) 493, 195 (2013).

[4] M. J. R. Heck, J. F. Bauters, M. L. Davenport, J. K. Doylend, S. Jain, G. Kurczveil, S. Srinivasan, Y. Tang, and J. E. Bowers, Hybrid silicon photonic integrated circuit technology, IEEE J. Sel. Top. Quantum Electron. 19, 6100117 (2013).

[5] D. Brunner and I. Fischer, Reconfigurable semiconductor laser networks based on difractive coupling, Opt. Lett. 40, 3854 (2015).

[6] J. Shena, J. Hizanidis, V. Kovanis, and G. P. Tsironis, Turbulent chimeras in large semiconductor laser arrays, Sci. Rep. 7, 42116 (2017).

[7] S. Osher and J. A. Sethian, Fronts propagating with curvaturedependent speed: Algorithms based on Hamilton-Jacobi formulations, J. Comput. Phys. 79, 12 (1988).

[8] S. Osher and R. Fedkiw, Level Set Methods and Dynamic Implicit Surfaces (Springer, New York, 2003).

[9] O. Miller, Photonic design: From fundamental solar cell physics to computational inverse design, Ph.D. thesis, University of California, Berkeley, 2013.

[10] A. Y. Piggott, J. Lu, K. G. Lagoudakis, J. Petykiewicz, T. M. Babinec, and J. Vukovic, Inverse design and demonstration of a compact and broadband on-chip wavelength demultiplexer, Nat. Photonics 9, 374 (2015).

[11] M. P. Bendsoe and O. Sigmund, Topology Optimization: Theory, Methods, and Applications (Springer, Heidelberg, 2003).

[12] M. P. Bendsoe, Generating optimal topologies in structural design using a homogenization method, Comput. Methods Appl. Mech. Eng. 71, 197 (1988).

[13] M. Gustafsson and S. Nordebo, Optimal antenna currents for Q, superdirectivity, and radiation patterns using convex optimization, IEEE Trans. Antennas Propag. 61, 1109 (2013).

[14] M. Gustafsson, C. Sohl, and G. Kristensson, Physical limitations on antennas of arbitrary shape, Proc. R. Soc. London, Ser. A 463, 2589 (2007).

[15] C. Khandekar, W. Jin, O. D. Miller, A. Pick, and A. W. Rodriguez, Giant frequency-selective near-field energy transfer in active-passive structures, Phys. Rev. B 94, 115402 (2016).

[16] D. Liu, L. H. Gabrielli, M. Lipson, and S. G. Johnson, Transformation inverse design, Opt. Express 21, 14223 (2013).

\section{ACKNOWLEDGMENTS}

This work was partially supported by the Ministry of Education and Science of the Republic of Kazakhstan via Contract No. 339/76-2015 and Nazarbayev University ORAU grant (No. 20162031) "Structured light for nonlinear and topological photonics". The authors would like to thank Dr. A. Nachman for bringing to their attention the utility of level-set methods.
[17] O. Hess and E. Scholl, Spatio-temporal dynamics in twin-stripe semiconductor lasers, Phys. D (Amsterdam, Neth.) 70, 165 (1994)

[18] O. Hess and E. Scholl, Eigenmodes of the dynamically coupled twin-stripe semiconductor laser, Phys. Rev. A 50, 787 (1994).

[19] E. Kapon, J. Katz, and A. Yariv, Supermode analysis of phaselocked arrays of semiconductor lasers, Opt. Lett. 9, 125 (1984).

[20] C. A. Valagiannopoulos and A. Alù, The role of reactive energy in the radiation by a dipole antenna, IEEE Trans. Antennas Propag. 63, 3736 (2015).

[21] C. A. Balanis, Advanced Engineering Electromagnetics (Wiley, New York, 1989), pp. 606-608.

[22] C. A. Valagiannopoulos, A novel methodology for estimating the permittivity of a specimen rod at low radio frequencies, J. Electromagn. Waves. Appl. 24, 631 (2010).

[23] C. A. Valagiannopoulos, N. L. Tsitsas, and G. Fikioris, Convergence analysis and oscillations in the method of fictitious sources applied to dielectric scattering problems, J. Opt. Soc. Am. A 29, 1 (2012).

[24] M. Abramowitz and I. A. Stegun, Handbook of Mathematical Functions (National Bureau of Standards, Washington D.C., 1970), pp. 360-361.

[25] C. A. Valagiannopoulos, N. L. Tsitsas, and A. H. Sihvola, Unlocking the ground: Increasing the detectability of buried objects by depositing passive superstrates, IEEE Trans. Geosci. Remote Sens. 54, 3697 (2016).

[26] D. S. Jones, Theory of Electromagnetism (Pergamon, Oxford, 1964), pp. 269-271.

[27] E. W. Montroll and R. B. Potts, Effect of defects on lattice vibrations, Phys. Rev. 100, 525 (1955).

[28] T. Erneux, V. Kovanis, A. Gavrielides, and P. M. Alsing, Mechanism for period-doubling bifurcation in a semiconductor laser subject to optical injection, Phys. Rev. A 53, 4372 (1996).

[29] Mathworks, rcond documentation, https://www.mathworks. com/help/matlab/ref/rcond.html

[30] S. Lang, Linear Algebra (Springer, New York, 1970).

[31] Mathworks, Fast and accurate symmetric positive definite matrix inverse using Cholesky decomposition, https://www. mathworks.com/matlabcentral/fileexchange/34511-fast-andaccurate-symmetric-positive-definite-matrix-inverse-usingcholesky-decomposition

[32] A. Singh, R. Dehiya, P. K. Gupta, and M. Israil, A MATLAB based 3D modeling and inversion code for MT data, Comput. Geosci. 104, 1 (2017).

[33] S. T. M. Fryslie, Z. Gao, H. Dave, B. J. Thompson, K. Lakomy, S. Lin, P. Decker, D. McElfresh, J. E. Schutt-Aine, and K. D. Choquette, Modulation of coherently-coupled phased photonic crystal vertical cavity laser arrays, IEEE J. Sel. Top. Quantum Electron. 99, 1 (2017). 